\title{
Clustering in Interfering Binary Mixtures
}

\author{
Sarah Miracle ${ }^{\star}$, Dana Randall ${ }^{\star \star}$, and Amanda Pascoe Streib $b^{\star \star \star}$ \\ Georgia Institute for Technology, Atlanta GA 30332, USA \\ \{sarah.miracle@cc, randall@cc, apascoe3@math\}.gatech.edu
}

\begin{abstract}
Colloids are binary mixtures of molecules with one type of molecule suspended in another. It is believed that at low density typical configurations will be well-mixed throughout, while at high density they will separate into clusters. We characterize the high and low density phases for a general family of discrete interfering binary mixtures by showing that they exhibit a "clustering property" at high density and not at low density. The clustering property states that there will be a region that has very high area to perimeter ratio and very high density of one type of molecule. A special case is mixtures of squares and diamonds on $\mathbb{Z}^{2}$ which corresond to the Ising model at fixed magnetization.
\end{abstract}

Keywords: discrete colloids, Ising model, phase separation, Peierls argument, equilibrium distribution.

\section{Introduction}

Colloids are mixtures of two types of molecules in suspension where all nonoverlapping arrangements are equally likely. When the density of each type of molecule is low, the mixtures are homogeneous and consequently exhibit properties that make them suitable for many industrial applications, including fogs, gels, foods, paints, and photographic emulsions (see, e.g., 1], 11]). In contrast, when the density is high, the two types of molecules separate whereby one type appears to cluster together. Although this behavior is similar to phase transitions that occur in other discrete models, such as the Ising and Potts models, here the two types of molecules do not possess any enthalpic forces causing like particles to attract or disparate particles to repel. In contrast, the behavior of colloids is purely entropic - the only restriction is a "hard-core" constraint requiring objects to remain in non-overlapping positions, and clustering occurs at high density because the overwhelming majority of configurations in the stationary distribution are believed to exhibit such a separation. While the experimental study of colloids is pervasive in surface chemistry, material science, physics, and nanotechnology, there has been little rigorous work explaining their behavior.

\footnotetext{
* Supported in part by NSF CCF-0830367 and a DOE Office of Science Graduate Fellowship.

** Supported in part by NSF CCF-0830367 and CCF-0910584.

$\star \star \star$ Supported in part by a National Physical Sciences Consortium Fellowship, a Georgia Institute of Technology ACO Fellowship and NSF CCF-0910584.
} 
Even running simulations has been challenging because local algorithms will be slow to converge at high density. Dress and Krauth 7] introduced an algorithm to try to overcome this obstacle, but this too was shown to require time exponential in the number of molecules in some cases [14. Nonetheless, their algorithm seems to be well-behaved in practice, and Buhot and Krauth 3] provided simulations showing strong heuristic evidence of the presence of two distinct phases in colloid models consisting of different sized squares.

Frenkel and Louis 9] studied an interesting discrete model of colloids whose behavior can be related to the Ising model, a standard model of ferromagnetism. Their model consists of mixtures of unit squares in a region of $\mathbb{Z}^{2}$ and diamonds of area $1 / 2$ that sit on lattice edges (see Fig. 1). They show that this colloid model, which we call Model 1, corresponds to an Ising model, where the density of squares fixes the magnetization and the density of diamonds determines the temperature (see Section 2.1). The Ising model at low temperature is known to exhibit clustering of positive spins. In fact the precise limiting shape of the cluster known as the Wulff shape has been extensively studied using sophisticated techniques (see, e.g. [5], or the references therein). Model 1 then inherits the phase transition arising in the Ising model which shows there will be clustering at high densities [13. In this paper we study clustering using elementary methods that apply to a large class of natural colloid models. We characterize clustering directly in terms of the parameters arising from the model to distinguish between the high and low phases and understand the role the density of each type of molecule plays.

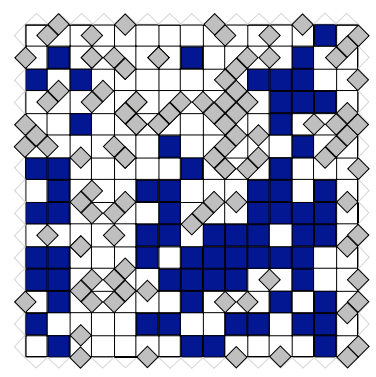

Fig. 1. Model 1, squares and diamonds on the $n \times n$ grid $L_{n}$

We consider a class of interfering binary mixtures. Let $\left(\Lambda_{A}, \Lambda_{B}\right)$ be a pair of planar lattices such that a face of $\Lambda_{A}$ and a face of $\Lambda_{B}$ are either disjoint, intersect at a single vertex, or intersect at a simply-connected region that is isomorphic to a fixed shape $s$ with nonzero area. For example, in Model 1, $\Lambda_{A}$ is the Cartesian lattice $\mathbb{Z}^{2}$ and $\Lambda_{B}$ is the set of diamonds bisected by edges in $\mathbb{Z}^{2}$; then $s$ is an isosceles triangle with unit base and height $1 / 2$ (Fig. (1). We consider the intersection of these lattices with some finite region $L$, where $L_{A}=\Lambda_{A} \cap L$ and $L_{B}=\Lambda_{B} \cap L$. We are given a set of tiles; $A$-tiles lie on the faces of $L_{A}$ and $B$-tiles lie on the faces of $L_{B}$ with the additional requirement that tiles must not overlap. In Section 5, we will give examples of other interfering binary mixtures, 
including independent sets, that arise naturally in combinatorics and statistical physics and contrast these with a non-interfering binary mixture that provably does not exhibit clustering.

It is often useful to switch from a model where the number of tiles of each type are fixed to a so-called grand-canonical ensemble where these are allowed to vary. Here, however, typical configurations would have a preponderance of only one type of tile at most high densities and the balanced configurations we are interested in would be exponentially unlikely. Instead, we fix the number of $A$ tiles and allow the $B$-tiles to vary stochastically. Each configuration $\sigma$ has weight proportional to $\lambda^{d(\sigma)}$, where $d(\sigma)$ is the number of $B$-tiles in $\sigma$. The choice of $\lambda$ controls the expected density of $B$-tiles.

Our goal now is to understand when clustering occurs in terms of the (expected) density of each type of tile. First we define a clustering property for configurations of tiles. Informally we have clustering if there exists a dense region $R$ in $\Lambda_{A}$ with $\Omega\left(n^{2}\right)$ area and $O(n)$ perimeter. Our main theorems demonstrate that at high density interfering binary mixtures exhibit the clustering property while at low densities they do not. We give precise definitions of the clustering property and state the main theorems in Section 2, In Sections 3 and 4 we prove the two main theorems in the context of Model 1 and in Section 5 we explain the generalization to other interfering binary mixtures.

The key tools in our proofs are careful Peierls arguments, used in statistical physics to study uniqueness of the Gibbs state and phase transitions (see, e.g., [4, 6]), and in computer science to study slow mixing of Markov chains (see, e.g., 2 [, 10, [15]). Peierls arguments allow you to add and remove contours by complementing the interiors of those contours. The main challenge here is maintaining the number of $A$-tiles, making the arguments considerably more difficult. We introduce the concept of bridge systems, to handle multiple contours by connecting components and make it possible to efficiently encode the boundaries of all contours removed. The encoding is necessary to account for the entropy/energy tradeoffs in these maps.

\section{Binary Mixtures and the Clustering Property}

We begin by formalizing the model, defining clustering and stating our main theorems.

\subsection{Interfering Binary Mixtures}

Recall $A$-tiles lie on faces of $L_{A}=\Lambda_{A} \cap L$ and $\left|L_{A}\right|$ is the total number of faces of $L_{A}$. Given constants $\lambda>1$, and $0<b<1 / 2$, where $b\left|L_{A}\right| \in \mathbb{Z}$, define $\Omega=\Omega(b, \lambda)$ as the set of non-overlapping packings of $L$ with $b\left|L_{A}\right|$ A-tiles and any number of B-tiles (where a tile can only be placed on a face of its type). We wish to study the distribution $\pi(\rho)=\lambda^{d(\rho)} / Z$, where $d(\rho)$ is the number of B-tiles in $\rho$ and $Z=\sum_{\rho \in \Omega} \lambda^{d(\rho)}$ is a normalizing constant. Our goal is to determine whether a configuration chosen according to $\pi$ is likely to have clusters of $A$-tiles. 
In Sections 21-4, we study Model 1, and in Section 5, we generalize the techniques to other models of interfering binary mixtures. We start by defining the Ising model on the $n \times n$ grid $L_{n}$ and explaining the equivalence with Model 1. Let $\bar{G}=(\bar{V}, \bar{E})$ be the dual lattice region to $L_{n}$ and let $\rho \in\{+,-\}^{\bar{V}}$ be an assignment of spins to each of the vertices in $\bar{V}$ (i.e., the faces in $V$ ). The weight of a configuration is $\bar{\pi}(\rho)=e^{\beta\left|\bar{E}_{d}(\rho)\right|} / \bar{Z}$, where $\bar{E}_{d}(\rho) \subseteq \bar{E}$ is the set of edges in $\bar{G}$ whose endpoints have different spins in $\rho, \beta$ is inverse temperature and $\bar{Z}$ is the normalizing constant.

For Model 1, given a configuration $\rho$ in $\Omega$, let the square structure $\Gamma(\rho)$ be the configuration $\sigma$ obtained from $\rho$ by removing all of its $B$-tiles (diamonds). We consider the set $\widehat{\Omega}$ of all such square structures with $b n^{2} A$-tiles (squares). Let $\widehat{\pi}$ be the induced distribution on $\widehat{\Omega}$; that is, for $\sigma \in \widehat{\Omega}$, let $\widehat{\pi}(\sigma)=\sum_{\rho \in \Gamma^{-1}(\sigma)} \pi(\rho)$. For $\sigma$ in $\Omega$ or $\widehat{\Omega}$, define the perimeter of $\sigma$ to be the edges that belong to exactly one $A$-tile in $\sigma$, and define $\kappa(\sigma)$ as the length of the perimeter of $\sigma$. Let $e(\sigma)$ be the number of edges that are not incident to any $A$-tile in $\sigma$. We find that

$$
\widehat{\pi}(\sigma)=\sum_{k=0}^{e(\sigma)} \frac{\lambda^{k}}{Z}\left(\begin{array}{c}
e(\sigma) \\
k
\end{array}\right)=\frac{1}{Z}(1+\lambda)^{e(\sigma)}=(1+\lambda)^{2 n^{2}-2 b n^{2}} \frac{\mu^{\kappa(\sigma)}}{Z},
$$

where $\mu=(1+\lambda)^{-\frac{1}{2}}$. Thus, the total perimeter of the square structure completely determines the probability that it will show up in $\Omega$. This directly implies the equivalence with the Ising Model: give a face $f$ of $L_{n}$ a positive spin if there is an $A$-tile on $f$ and a negative spin otherwise. Since the weight of a configuration is determined exactly by the number of edges with opposite spins in $L_{n}$, this is the Ising model with a fixed number of positive spins for some $\lambda$ that is a function of $\beta$, known as fixed magnetization.

\subsection{The Clustering Property}

The goal of this paper is to show that when the density of $B$-tiles is high, interfering binary mixtures cluster, while at low density they do not. First, we characterize clustering in this context. Intuitively, a configuration has the clustering property if there is a large region densely filled with $A$-tiles. More precisely, let a region $R$ be any set of faces in $L_{n}$. Its perimeter, $\kappa(R)$ is the number of edges adjacent to a face in $R$ and a face in $\bar{R}=L_{n} \backslash R$. Let $c=\min \left\{\frac{b}{2}, \frac{1}{100}\right\}$.

Definition 1. We say that a configuration $\sigma \in \Omega($ or $\Gamma(\sigma) \in \widehat{\Omega})$ has the clustering property if it contains a region $R$ which satisfies the following properties:

1. $R$ contains at least $(b-c) n^{2}$ A-tiles,

2. the perimeter of $R$ is at most $8 \sqrt{b} n$, and

3. the density of A-tiles in $R$ is at least $1-c$ and in $\bar{R}$ is at most $c$.

If a configuration has the clustering property, we show that it contains an $n^{1 / 3} \times$ $n^{1 / 3}$ window with high density and one with low density, demonstrating the heterogeneity of the configuration. In Section 5.2 we contrast this with Model 4, related to bond percolation, which remains homogeneous at all densities. 


\subsection{Main Results}

We show that at high density interfering binary mixtures have the clustering property while at low densities they do not. Specifically, we prove the following theorems in the context of Model 1 on the $n \times n$ region $L_{n}$ with $b n^{2} A$-tiles and the density of $B$-tiles determined by $\lambda$. In Section [5, we show they also hold for other interfering binary mixtures.

Theorem 1. For $0<b \leq 1 / 2$, there exist constants $\lambda^{*}=\lambda^{*}(b)>1, \gamma_{1}<1$ and $n_{1}=n_{1}(b)$ such that for all $n>n_{1}, \lambda \geq \lambda^{*}$ a random sample from $\Omega$ will have the clustering property with probability at least $\left(1-\gamma_{1}{ }^{n}\right)$.

Theorem 2. For $0<b<1 / 2$, there exist constants $\lambda_{*}=\lambda_{*}(b)>0, \gamma_{2}<1$ and $n_{2}=n_{2}(b)$ such that for all $n>n_{2}, \lambda \leq \lambda_{*}$ a random sample from $\Omega$ will not have the clustering property with probability at least $\left(1-\gamma_{2}{ }^{n}\right)$.

Furthermore, it follows from the proofs that at low density if a dense region $R^{\prime}$ has area $\Omega\left(n^{2}\right)$ then it must have perimeter $\Omega\left(n^{2}\right)$. Notice that in the case $b>1 / 2$ we can obtain comparable results by the symmetry of the $A$-tiles to the empty space. Indeed, in this case if $\lambda$ is sufficiently high we will see empty cells clustering within a sea of $A$-tiles and for low density the empty cells will be well-distributed.

Note that since clustering is just a property of the $A$-tiles, it suffices to prove Theorems 1 and 2 for weighted square structures $\widehat{\Omega}$, involving just the $A$-tiles. From this point we focus on $\widehat{\Omega}$, and we refer to $A$-tiles just as tiles.

\section{High Density of $B$-tiles}

We concentrate first on interfering binary mixtures at high density to prove Theorem 1 Define $\Psi \subset \widehat{\Omega}$ to be the set of configurations that have the clustering property; then we show that $\widehat{\pi}(\widehat{\Omega} \backslash \Psi) \leq \gamma_{1}^{n} \widehat{\pi}(\Psi)$ for some constant $\gamma_{1}<1$. To achieve this, we apply a Peierls argument, in which we define a map $f: \widehat{\Omega} \backslash \Psi \rightarrow \Psi$ and show that for all $\tau \in \Psi$,

$$
\sum_{\sigma \in f^{-1}(\tau)} \widehat{\pi}(\sigma) \leq \gamma_{1}^{n} \widehat{\pi}(\tau)
$$

Given a configuration $\sigma \in \widehat{\Omega} \backslash \Psi$, the map $f$ removes a large set $T$ of tiles in $\sigma$ and reassembles them in a single large component in $f(\sigma)$. This decreases the total perimeter of the configuration significantly, and therefore $\widehat{\pi}(f(\sigma))$ is exponentially larger than $\widehat{\pi}(\sigma)$. The challenge is to bound the number of configurations that map to a given $\tau \in \Psi$ by carefully encoding the preimages of $\tau$.

Some definitions will be helpful. We say two tiles are adjacent if their borders share an edge. A component is a maximal connected set of tiles, and maximal connected segments of the perimeter of $\sigma$ are contours. The set $T$ of tiles we remove will be a union of components, which we identify using a system of 
"bridges" connecting these components (Fig. 2). The key is that the number of edges in the bridges is at most a constant times the total perimeter of the components bridged. Then if $E$ is the set of all edges in bridges or along contours bridged, we can bound $\left|f^{-1}(\tau)\right|$ by the number of ways that those $E$ edges could be distributed in $\sigma$. Finally, we show that there is a sparse, roughly square region in the resulting configuration where we can add the $T$ tiles. We complement that region to obtain $f(\sigma)$.

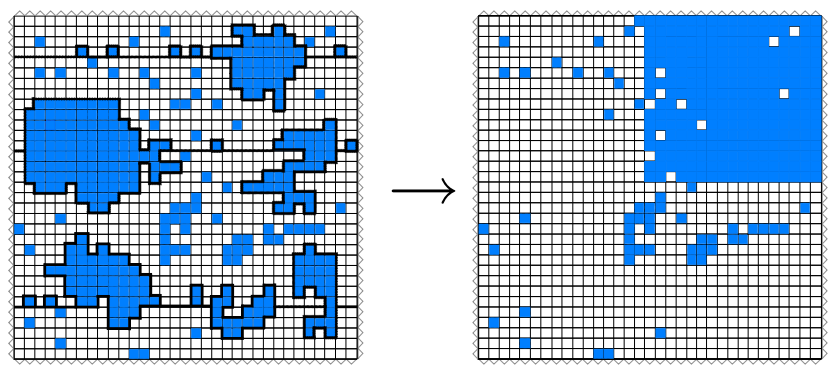

Fig. 2. A configuration $\sigma \in \widehat{\Omega} \backslash \Psi$ and the image $f(\sigma)$ of $\sigma$ in $\Psi$

Building Bridges. Given a region $R$, let $C(R)$ be the set of contours fully contained within the interior of $R$ and define the outer contours to be those in $C(R)$ that are not contained in the interior of other contours in $C(R)$. The interior of the outer contours of components are called holes and the interior of the outer contours of holes are called islands.

Consider first the case in which there are no components with holes. Suppose $B$ is a set of edges of $L_{n}$ connecting some subset $S$ of the contours to the boundary of $L_{n}$. We call $B$ a set of bridges and $S$ a set of bridged contours. A cell in $L_{n}$ or a tile is called unbridged if it is not bounded by a bridged contour. Then $(B, S)$ is a $c$-bridge system for $\sigma \in \widehat{\Omega}$ if the number of unbridged tiles is at most $c$ times the number of unbridged cells, and $|B| \leq(1-c) /(2 c) \kappa(S)$. If $\sigma$ has components with holes, then first construct a $c$-bridge system $(B, S)$ for $\sigma^{\prime}$, obtained from $\sigma$ by filling all the holes. Next for each bridged contour $X$ in $\sigma$, construct a $c$-bridge system for the region bounded by $X$ (treating tiles as empty cells and empty cells as tiles). Recurse until you obtain $c$-bridge systems for each bridged contour at every level of the recursion. We call this a $c$-bridge system of $\sigma$. We defer the details to the full version of the paper.

Lemma 1. There exists a c-bridge system for any configuration $\sigma \in \widehat{\Omega}$.

Proof. We may assume that $\sigma$ has no holes, since otherwise we recurse as described above. Now we use induction on the number of contours in $R$. If there are no contours, then clearly $(\emptyset, \emptyset)$ is a $c$-bridge system for $R$. Otherwise, define $t(R)$ to be the tiles in $R$, and $x(R)$ is the number of empty cells in $R$. Let $\mathbb{H}$ be the set of horizontal lines through $R$. For every $H \in \mathbb{H}$, if $|t(R) \cap H|\langle c|R \cap H|$ then we are done, since then $(\emptyset, \emptyset)$ is a $c$-bridge system for $R$. Otherwise there exists 
a horizontal line $H$ such that $|t(R) \cap H| \geq c|R \cap H|$. Then let $B$ be the set of bottom edges of every outer cell in $H \cap R$. See Fig. 3, where the dark black edges along the line $H$ are the new bridges. Let $S$ be the set of contours connected in this step. We know that $\kappa(S) \geq 2|t(R) \cap H| \geq 2 c|R \cap H| \geq 2 c /(1-c)|x(R) \cap H|$, so $|B| \leq(1-c) /(2 c) \kappa(S)$. We obtain $R^{\prime}$ from $R$ by removing the cells bounded by a contour in $S$, as in Fig. 3. Then by induction, there exists a $c$-bridge system $\left(B^{\prime}, S^{\prime}\right)$ of $R^{\prime}$. Then $\widehat{B}:=B \cup B^{\prime}$ is a set of bridges connecting the contours in $\widehat{S}=S \cup S^{\prime}$ to each other and to the boundary of $R$. Moreover, $|\widehat{B}| \leq \frac{1-c}{2 c} \kappa(\widehat{S})$ and the number of unbridged tiles is at most $c$ times the number of unbridged cells. Hence $(\widehat{B}, \widehat{S})$ is a $c$-bridge system for $R$.

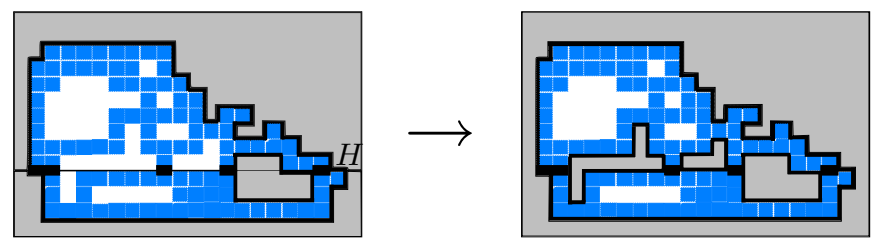

Fig. 3. Before and after one step of the construction of a $c$-bridge system for a region $R$; the solid lighter grey area is exterior to $R$

Once we have a $c$-bridge system, we can apply a map in which we complement an entire region of cells, making tiled cells empty and vice versa. This map significantly reduces the perimeter, but can dramatically change the total number of tiles. Recall we must maintain the total number of tiles, so we may need to supplement by adding extra tiles from another region or we may have extra tiles, which we will put in our "bank" for later. At the end of the process we will find a roughly square region that we can again complement using the bank of extra tiles so that the total number of tiles is restored to $b n^{2}$ at minimal cost.

Finding a Sparse Box. We now show that after removing all but $c n^{2}$ tiles, there exists a region of low density where we can place the tiles in our bank.

Lemma 2. For $(b-c) n^{2} \leq a<b n^{2}$, there exists a constant $n_{3}=n_{3}(b)$ such that for all $n \geq n_{3}$, if $\rho$ is a configuration with at most $c n^{2}$ tiles then $\rho$ contains a roughly square region $R^{\prime}$ such that complementing $R^{\prime}$ requires a additional tiles and the change in total perimeter is at most $5 \sqrt{a}$.

Proof. Given a region $R$, let $d(R)$ denote the number of tiles needed to complement $R$; this is exactly the area of $R$ minus twice the number of tiles in $R$. Let $l=\lceil\sqrt{8 a / 7}\rceil$. First we show that there exists a square $l \times l$ region $R$ such that $d(R) \geq a$. Assume that such a square does not exist. Divide the grid into $\left\lfloor\frac{n}{l}\right\rfloor^{2}$ disjoint squares with side length $l$ and consider any square. Let $t$ be the number of tiles in the square. The empty volume is at least $l^{2}-t$. By assumption each square satisfies $l^{2}-t<t+a$, and so $t>\frac{l^{2}-a}{2}$. In particular, $8 a / 7 \leq l^{2}<a+2 c n^{2}$, 
so we may assume that $a<14 c n^{2}$. This implies that $l \leq \sqrt{8 a / 7}+1 \leq 1+4 \sqrt{c} n$. However, if $T$ is the total number of tiles,

$$
c n^{2} \geq T>\left\lfloor\frac{n}{l}\right\rfloor^{2} \frac{l^{2}-a}{2} \geq \frac{n^{2}}{2}\left(1-\frac{l}{n}\right)^{2}\left(1-\frac{a}{l^{2}}\right)>\frac{n^{2}\left(1-\frac{1}{n}-4 \sqrt{c}\right)^{2}}{16} \geq c n^{2},
$$

since $c \leq \frac{1}{65}$ and $n \geq n_{3}$, a contradiction. Therefore there exists an $l \times l$ square $R$ such that $d(R) \geq a$. Remove cells from $R$ one at a time, starting with the bottom row of $R$ and moving across, until we obtain a region $R^{\prime} \subseteq R$ with $d\left(R^{\prime}\right)=a$. This can be done because removing one cell at a time changes $d$ by at most 1 . This region $R^{\prime}$ is roughly square and has perimeter at most $4 \sqrt{8 a / 7}<5 \sqrt{a}$.

The Proof of Theorem 1, Finally we can prove Theorem 1, showing that for large $\lambda$ a typical configuration will have the clustering property.
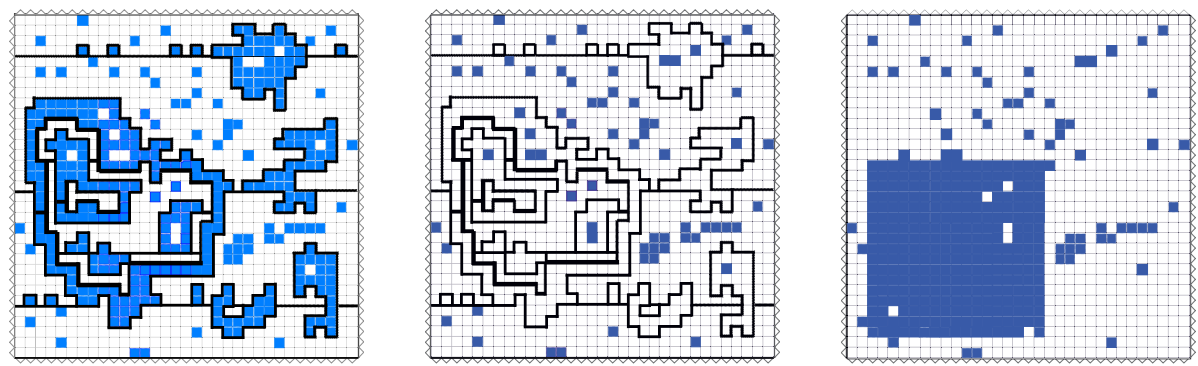

Fig. 4. A $c$-bridge system for $\sigma \in \widehat{\Omega} \backslash \Psi$; the image $f_{1}(\sigma)$; and $f(\sigma)=f_{2} \circ f_{1}(\sigma)$

Proof of Theorem 11. Let $\sigma \in \widehat{\Omega} \backslash \Psi$. Construct a $c$-bridge system $(B, S)$ for $L_{n}$ as described in Lemma 1. That is, $(B, S)$ is a set of bridges in $L_{n}$ connecting some of the components, some of the holes within those components, some of the islands within those holes, etc. For any bridged contour $X$, let $r(X)$ be the region bounded by $X$. If $r(X)$ is a component with holes, then we remove all outer tiles of $r(X)$ and complement all unbridged holes in $X$, using a subset of the tiles removed to fill in the holes. If $r(X)$ is a hole with islands, then we leave all of the unbridged islands alone. At this point, after complementing some number of regions, we have a bank of extra tiles; let $a$ be the number of tiles in the bank. Notice that by the definition of a $c$-bridge system, the density of tiles remaining is at most $c$, so $a \geq(b-c) n^{2}$.

Let $f_{1}(\sigma)$ be obtained from $\sigma$ by removing the bridged components and complementing as described above. Let $\mathcal{F}_{1}$ be the image of $f_{1}$ on $\widehat{\Omega} \backslash \Psi$; note that $\mathcal{F}_{1} \not \subset \widehat{\Omega}$ since the configurations in $\mathcal{F}_{1}$ have too few tiles. Let $\kappa$ be the total perimeter of all contours bridged. Then for any $\rho \in \mathcal{F}_{1}$, we claim that the number of preimages of $\rho$ whose bridged contours have total perimeter $\kappa$ is at most $5^{c_{3}}$ for $c_{3}=\left(1+\frac{1-c}{2 c}+\frac{1}{c_{2}}\right) \kappa$. Consider the $c$-bridge system obtained above for 
$L_{n}$. Let $V$ denote the leftmost vertical edges of the region. Let $S^{\prime}=S \cup V$. We perform what is essentially a depth-first-search traversal of the bridge system on $S^{\prime}$, starting at the top left corner of $L_{n}$. As we traverse an edge we record what type of edge it was using five bits that represent forward, left, right, bridge east, or bridge west (see full version for details). Given the encoded information, there is a unique way to distribute the contours. Hence for all perimeters $\kappa \geq c_{2} n$ the number of preimages of $\rho$ whose bridged contours have total perimeter $\kappa$ is at most $5^{|B|+\kappa+n} \leq 5^{c_{3}}$. Therefore $\left|f_{1}^{-1}(\rho)\right| \leq \sum_{\kappa \geq c_{2} n} 5^{c_{3}}$.

Let $\rho \in \mathcal{F}_{1}$ with $b n^{2}-a$ tiles. Lemma 2 shows how to find a region $S^{\prime}$ in $\rho$ to complement using the $a$ tiles from the bank to obtain $\tau$ in such a way that $\kappa(\tau)-\kappa(\rho) \leq 5 \sqrt{a}$. Let $f_{2}(\rho)=\tau$ and $f=f_{2} \circ f_{1}$. We can encode the boundary of $S^{\prime}$ with $n^{2} 3^{\kappa\left(S^{\prime}\right)} \leq n^{2} 3^{5 \sqrt{a}}$ information. Hence for any $\tau \in \Psi$,

$$
\left|f^{-1}(\tau)\right| \leq n^{2} 3^{5 \sqrt{a}} \max _{\rho \in f_{2}^{-1}(\tau)}\left|f_{1}^{-1}(\rho)\right| .
$$

Let $\sigma \in \widehat{\Omega} \backslash \Psi$, and as above let $\kappa$ be the total perimeter of components bridged in $\sigma$ (recall $\kappa(\sigma)$ is the total perimeter of all contours in $\sigma$ ). If $\kappa \leq 8 \sqrt{a}$, then $\sigma \in \Psi$, a contradiction. To see this, define the parity of a cell to be 1 if it is contained within an odd number of bridged contours and 0 otherwise, and let $R$ be the set of cells with parity 1 . Then $R$ has density at least $1-c$, perimeter at most $8 \sqrt{a}$ and $a \geq(b-c) n^{2}$ tiles. Moreover, $\bar{R}$ has density at most $c$. Thus $R$ is the region we require, and so $\sigma \in \Psi$. This implies $\kappa>8 \sqrt{a}$. We have shown that $\kappa(\sigma)-\kappa(f(\sigma))>\kappa-5 \sqrt{a}>\kappa / 4$. Let $\tau \in \Psi$ and

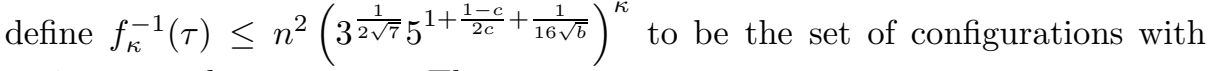
perimeter $\kappa$ that map to $\tau$. Then

$$
\pi(\tau)^{-1} \sum_{\sigma \in f^{-1}(\tau)} \pi(\sigma) \leq \sum_{\sigma \in f^{-1}(\tau)} \mu^{\kappa(\sigma)-\kappa(f(\sigma))} \leq \sum_{\kappa=8 \sqrt{a}}^{2 n^{2}} \mu^{\kappa / 4}\left|f_{\kappa}^{-1}(\tau)\right| \leq \gamma_{1}^{n},
$$

for some $\gamma_{1}<1$, if $\mu \leq \mu^{*}<\left(3^{\frac{1}{2 \sqrt{7}}} 5^{1+\frac{1-c}{2 c}+\frac{1}{16 \sqrt{b}}}\right)^{-4}$. Thus the theorem holds if $\lambda \geq \lambda^{*}=\mu^{*-2}-1$.

As a corollary, we find that if a configuration has the clustering property then there exists an $n^{1 / 3} \times n^{1 / 3}$ window with high density and one with low density. We defer this straightforward proof to the full version of the paper.

Corollary 1. For $0<b \leq 1 / 2$ there exists a constant $n_{4}=n_{4}(b)$ such that for all $n>n_{4}$, if $\sigma$ satisfies the clustering property then $\sigma$ contains square $n^{1 / 3} \times n^{1 / 3}$ windows $W_{1}$ and $W_{2}$ such that the density of tiles in $W_{1}$ is at least $.99(1-c)$ and the density of tiles in $W_{2}$ is at most $2.1 \mathrm{c}$.

\section{Low Density of $B$-tiles}

We now examine the low density case and prove Theorem 2, stating that typical configurations will not have the clustering property. For small enough $\lambda$, the 
$A$-tiles will be well-distributed throughout $L_{n}$, in the following sense. Any large dense region must have perimeter on the order of $n^{2}$.

Proof of Theorem 囵. Define $\delta=((1-c) /(b-c))^{b-c}$. Let $\Psi^{\prime} \subset \widehat{\Omega}$ be the set of configurations with a region $R$ that have density at least $1-c$, at least $(b-c) n^{2}$ tiles, and perimeter less than $\alpha n^{2}$, where $\alpha$ satisfies $0<\alpha<(\ln (\delta)-b \ln 2) /((1+$ $1 / c) \ln 5)$. We will show $\widehat{\pi}\left(\Psi^{\prime}\right)$ is exponentially small. Clearly $\Psi \subset \Psi^{\prime}$, so this implies that the clustering property is exponentially unlikely to occur.

For each $\sigma \in \Psi^{\prime}$, construct a $c$-bridge system for $\sigma$. As in the proof of Theorem 1, we complement all bridged components and all non-bridged holes within those components. We obtain $f_{1}(\sigma)$, which has $t_{\sigma} \leq c n^{2}$ tiles, and a bank of $a_{\sigma} \geq(b-c) n^{2}$ tiles. Next we define $N(\sigma)$ to be the set of all configurations obtained from $f_{1}(\sigma)$ by adding $a_{\sigma}$ tiles back at any empty location; then $|N(\sigma)|=\left(\begin{array}{c}n^{2}-t_{\sigma} \\ a_{\sigma}\end{array}\right)$. For each $\tau \in \widehat{\Omega}$, we need to bound the number of configurations $\sigma$ such that $\tau \in N(\sigma)$. As before, we can reconstruct the bridge system for $\sigma$ with $5^{\left(1+\frac{1-c}{2 c}\right) \kappa+n}$ information and we can recover the original with $2^{b n^{2}}$ information by recording whether each tile moved. Hence the number of $\sigma$ that map to $\tau$ is at most $5^{\left(1+\frac{1-c}{2 c}\right) \alpha n^{2}+n} 2^{b n^{2}} \leq\left(2^{b} \delta\right)^{n^{2} / 2}$ for large enough $n$.

Finally, we define a weighted bipartite graph $G\left(\Psi^{\prime}, \widehat{\Omega}, E\right)$ with an edge of weight $\pi(\sigma)$ between $\sigma \in \Psi^{\prime}$ and $\tau \in \widehat{\Omega}$ if $\tau \in N(\sigma)$. The total weight of edges is

$$
\sum_{\sigma \in \Psi^{\prime}} \pi(\sigma)|N(\sigma)| \geq \sum_{\sigma \in \Psi^{\prime}} \pi(\sigma)\left(\begin{array}{c}
n^{2}-\left(b n^{2}-a_{\sigma}\right) \\
a_{\sigma}
\end{array}\right) \geq \pi\left(\Psi^{\prime}\right) \delta^{-n^{2}} .
$$

However, the weight of the edges is at most $\sum_{\tau \in \widehat{\Omega}} \pi(\tau) \mu^{-4(b-c) n^{2}}\left(2^{b} \delta\right)^{n^{2} / 2}$. Let $\mu^{*}=\left(2^{b} / \delta\right)^{1 /(8(b-c))}$ and $\lambda^{*}=\left(\mu^{*}\right)^{-2}-1$. Thus for all $\mu<\mu^{*}$,

$$
\pi\left(\Psi^{\prime}\right)<\mu^{-4(b-c) n^{2}}\left(2^{b} \delta\right)^{n^{2} / 2} \delta^{-n^{2}}<\gamma_{2}^{n},
$$

for some $\gamma_{2}<1$, completing the proof.

\section{Other Models}

We conclude by considering other natural models of binary mixtures and showing that Theorems 1 and 2 still hold for the interfering models.

\subsection{Interfering Binary Mixtures}

Model 2: $A$-tiles are squares on $L_{n}$ and $B$-tiles are unit squares centered on vertices of $L_{n}$, (see Fig. 5(a)). It is not hard to see that this model corresponds exactly to an independent set model on the rotated grid where vertices correspond to the centers of $A$-tiles and $B$-tiles, and the number of even vertices is fixed. The number of odd vertices varies according to $\lambda$. Again the $A$-tiles will cluster together at high enough $\lambda$, leaving large regions to fill with $B$-tiles. 
The weight of a configuration $\sigma$ is proportional to $\lambda^{v}$, where $v$ is the number of vertices in $\sigma$ not intersecting any $A$-tiles (we call these open vertices). Hence we must argue that by removing several components and putting them together into a single large component, the number of open vertices increases. Indeed, the number of open vertices is proportional to the length of the perimeter, so this can be carried out. One must be careful, however, to define a component so that two tiles are adjacent if they share a vertex (not an edge). Otherwise, if a region looks like a checkerboard of tiles to empty space, and we remove every other row to create a new component, we decrease the perimeter but increase the number of occupied vertices. This cannot happen as long as we choose maximal connected subsets of tiles according to this definition of adjacency.

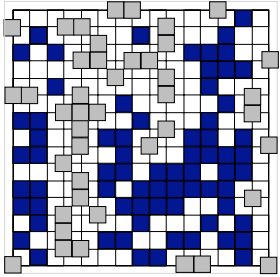

(a)

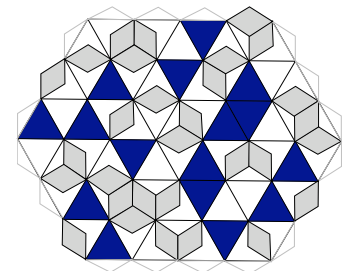

(b)

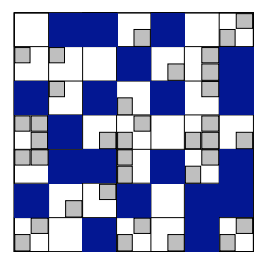

(c)

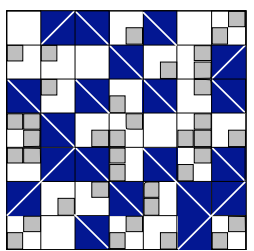

(d)

Fig. 5. (a) Model 2 (b) Model 3 (c) Model 4 (d) Model 4 and bond percolation

Model 3: $A$-tiles are triangles on the triangular lattice $\Lambda_{A}$ and $B$-tiles are lozenges bisected by edges of $\Lambda_{A}$, (see Fig. 5 (b)). Model 3 maps bijectively onto an Ising Model with fixed magnetization on $\Lambda_{A}$. In models like this, where the $A$-tiles are not square, the large component we create for Theorem 1 might not be square, but some other shape with large area to perimeter ratio, such as a hexagon in this context. The remaining details are similar.

\subsection{Noninterfering Binary Mixtures}

Model 4: $A$-tiles are unit squares on $L_{n}$ and $B$-tiles are squares of side length $1 / 2$ on the half-integer lattice, (see Fig. 5 (c)). This model is qualitatively different from the previous models since the placement of the $A$-tiles does not influence the number of places in which we can put the $B$-tiles. In fact, this model is just bond percolation on a rotated grid with a fixed number of edges, where we do not expect clustering at any density. To see the bijection, label a unit square with a Northwest-Southeast diagonal if it lies on an even face and label it with a Northeast-Southwest diagonal otherwise, as in Fig. [5(d). Notice that these lines form a subset of the edges of a rotated grid. If we have $b n^{2}$ $A$-tiles then each edge in the rotated grid is present with probability $b$. To illustrate the difference between the behavior of Model 4 and the interfering binary mixtures, consider an $n^{1 / 3} \times n^{1 / 3}$ window in each. In Model 4, the probability that any $n^{1 / 3} \times n^{1 / 3}$ box has density $d$ such that $d>1.5 b$ or $d<0.5 b$ is 
less than $\gamma_{3}^{n}$ for some constant $\gamma_{3}<1$ (see the full version for details). In contrast by Corollary 1, a configuration with the clustering property has a window with density $d \geq .99(1-c)$ and a window with density $d \leq 2.1 c$. Hence we see markedly different behavior between interfering and non-interfering binary mixtures.

\section{References}

1. Birdi, K.S.: Handbook of Surface and Colloid Chemistry. CRC Press, Boca Raton (2008)

2. Borgs, C., Chayes, J.T., Frieze, A., Kim, J.H., Tetali, P., Vigoda, E., Vu, V.H.: Torpid Mixing of Some MCMC Algorithms in Statistical Physics. In: 40th IEEE Symp. on Foundations of Computer Science (FOCS), pp. 218-229 (1999)

3. Buhot, A., Krauth, W.: Phase Separation in Two-dimensional Additive Mixtures. Phys. Rev. E 59, 2939-2941 (1999)

4. Dobrushin, R.L.: The Problem of Uniqueness of a Gibbsian Random Field and the Problem of Phase Transitions. Funct. Analysis and its App. 2, 302-312 (1968)

5. Dobrushin, R.L., Kotecký, R., Shlosman, S.B.: The Wulff Construction: A Global Shape from Local Interactions. American Mathematical Society, Providence (1992)

6. Dobrushin, R.L., Shlosman, S.: Constructive Criterion for the Uniqueness of Gibbs Fields. In: Statistical Physics and Dynamical Systems, pp. 347-370, Birhaurer, Basel (1985)

7. Dress, C., Krath, W.: Cluster Algorithm for Hard Spheres and Related Systems. J. Phys. A: Math. Gen. 28, L597 (1995)

8. Frenkel, D.: Order Through Disorder: Entropy-driven Phase Transitions. Lecture Notes in Physics, vol. 415, pp. 137-148 (1993)

9. Frenkel, D., Louis, A.: Phase Separation in Binary Hard-core Mixtures: An Exact Result. Physical Review Letters 68, 3363-3365 (1992)

10. Gore, V., Jerrum, M.: The Swendsen-Wang Algorithm Does Not Always Mix Rapidly. J. Statistical Phys. 97, 67-86 (1999)

11. Hiemenz, P., Rajagopalan, R.: Principles of Colloid and Surface Chemistry. CRC Press, Boca Raton (1997)

12. Krauth, W.: Cluster Monte Carlo algorithms. In: Hartmann, A.K., Rieger, H. (eds.) New Optimization Algorithms in Physics, Wiley-VCh, Chichester (2005)

13. Martinelli, F.: Lectures on Glauber dynamics for Discrete Spin Models. Lectures Notes in Math. vol. 1717, pp. 93-191 (1999)

14. Miracle, S., Randall, D., Streib, A.P.: Cluster Algorithms for Discrete Models of Colloids with Bars. In: 8th Workshop on Analytic Algorithmics and Combinatorics (ANALCO), pp. 135-150 (2011)

15. Randall, D.: Slow Mixing of Glauber Dynamics Via Topological Obstructions. In: 17th ACM-SIAM Symp. on Discrete Alg. (SODA), pp. 870-879 (2006)

16. Rodríguez-Guadarrama, L., Talsania, S., Mohanty, K., Rajagopalan, R.: Mixing Properties of Two-Dimensional Lattice Solutions of Amphiphiles. J. of Colloid and Interface Science 224, 188-197 (2001) 